\title{
Phase behaviour of mixtures of polyethylene glycol and polypropylene glycol: Influence of hydrogen bond clusters on the phase diagram
}

\author{
Susanne Eckert, ${ }^{a}$ Gerhard Meier $^{b}$ and Ingo Alig*a \\ a Deutsches Kunststoff-Institut, D-64289, Darmstadt, Schloßgartenstr. 6, Germany \\ ${ }^{b}$ IFF “Weiche Materie”, FZ-Jülich, Postfach 1913, D-52425, Jülich, Germany
}

Received 28th March 2002, Accepted 17th May 2002
First published as an Advance Article on the web 28th June 2002

The phase behaviour of a binary polymer blend of polyethylene glycol (PEG600) and polypropylene glycol (PPG1000) of low polydispersity was investigated. The coexistence curves of five different compositions, including the critical composition, were measured by differential refractometry. From the coexistence curves, the critical exponents of the order parameter could be determined. The order parameter of the coexistence curve of the critical composition was $\beta=0.357$, near to the Ising value. The measured coexistence curves for different compositions were different to each other and to the cloud point curve measured by small angle light scattering. These differences are explained by clusters built of intermolecular hydrogen bonds which lead to a broad effective molar mass distribution of the mixture. Two of the investigated mixtures, the mixture of critical composition ( $y_{\mathrm{PPG}}=0.46, y_{\mathrm{PPG}}$ : mass fraction of polypropylene glycol) and a mixture of non-critical composition $\left(y_{\mathrm{PPG}}=0.365\right)$ were also measured by static and dynamic light scattering. The measurements were carried out in the homogeneous one-phase region (above the critical temperature $T_{\mathrm{C}}$ ) and in the two-phase region in both coexisting phases. The light scattering measurements showed, additionally to the composition fluctuations due to the phase transition of demixing, a contribution of larger objects, which could be attributed to hydrogen bonded clusters. The size of these clusters was estimated to be $250 \mathrm{~nm}$ to $550 \mathrm{~nm}$.

\section{Introduction}

There have been many investigations on the phase behaviour of binary polymer mixtures in the last decades but systematic experimental studies on the influence of specific interactions like hydrogen bonds and the influence of the molar mass distribution on the phase diagram are rare.

On the theoretical side, the phase behaviour of binary polymer mixtures is described by the Flory-Huggins-Staverman theory. ${ }^{1-3}$ In this theory, specific interactions are incorporated empirically into the Flory-Huggins-Staverman-interaction parameter while the influence of the polydispersity of the polymers is neglected. The effects of polydispersity on the phase diagram have been studied theoretically (see e.g. ref. 4 and 5) but systematic experimental investigations are rare. There exist many measurements of cloud point curves of polymer mixtures, but not for coexistence curves. This is in contrast to low molecular weight mixtures where numerous measurements of the coexistence curves verify the 3d-Ising value of the critical exponent $\beta$ of the order parameter (see e.g. ref. 6). A measurement of the coexistence curve of a critical polymer mixture of polydimethylsiloxane and polyethylmethylsiloxane by differential refractometry ${ }^{7}$ yielded a critical exponent $\beta$ near the $3 \mathrm{~d}-$ Ising value. To our knowledge there is only one investigation of coexistence curves of non-critical polymer mixtures of $\mathrm{PEG}$ and $\mathrm{PPG}^{8}$ so far.

For binary polymer mixtures from monodisperse polymers, the coexistence curves are independent of the composition in the one-phase region of the mixture separating into two phases. Also the cloud point curve and the coexistence curves are expected to fall together for monodisperse mixtures. If the polydispersity of the system is high, these assumptions cannot be maintained, i.e. the coexistence curves depend on the mass fraction of the components in the one-phase region and are different from the cloud point curve.
The aim of this paper is to investigate the influence of hydrogen bonds on the phase behaviour of an oligomer blend of polyethylene glycol (PEG) and polypropylene glycol (PPG) with molecular weights of $M=600 \mathrm{~g} \mathrm{~mol}^{-1}$ (PEG600) and $M=1000 \mathrm{~g} \mathrm{~mol}^{-1}$ (PG1000), respectively. Both samples have a very narrow molar mass distribution. The ratio $M_{\mathrm{W}} / M_{\mathrm{N}}$ is 1.04 for PEG600 and 1.03 for PPG1000. Although the polydispersities of the two samples are very low, they can build large clusters by intermolecular hydrogen bonding due to their large amount of hydroxy groups. These clusters lead to an effective polydispersity of the mixture which influences the phase diagram. Because of this, the influence of hydrogen bonds on the phase diagram can be studied very well by the measurement of coexistence curves of different compositions of this mixture. The low viscosity of the mixtures allowed us to measure the coexistence curves with a differential refractometer.

In order to get a more direct indication for the clusters and an estimate of the cluster size we performed dynamic and static light scattering experiments in the PPG-rich phase, the PEGrich phase and in the one-phase region. The size of the clusters can be determined by dynamic light scattering measurements due to the diffusion of the clusters whereas the static light scattering yields direct information on the size from the static structure factor. This paper will focus on the phase diagram and the cluster size. The analysis of the light scattering process due to critical fluctuations near the phase transition of demixing will be the topic of a further paper which is in progress. ${ }^{9}$

The paper is organized as follows: After a survey of the theory of phase behaviour and light scattering of polymer mixtures (Section II A, B, and C), a sketch of the experimental conditions is given in Section III. The phase diagram of the system is described in Section IV A, the analysis of the static and dynamic light scattering data is depicted in Section IV B. The paper closes with some concluding remarks. 


\section{Theory}

\section{A. Phase behaviour of polymer blends}

1. Binary polymer mixtures of monodisperse components. The phase behaviour of binary polymer blends with monodisperse components is described by a lattice theory of Flory, ${ }^{1}$ Huggins, ${ }^{2}$ and Staverman and Van Santen. ${ }^{3}$ In this theory, the Gibbs energy of mixing is described by:

$$
\frac{\Delta_{\mathrm{M}} G_{N}}{k_{\mathrm{B}} T}=\frac{\phi_{1}}{N_{1}} \ln \phi_{1}+\frac{\phi_{2}}{N_{2}} \ln \phi_{2}+\chi_{\mathrm{FHS}} \phi_{1} \phi_{2},
$$

where $\Delta_{\mathrm{M}} G_{N}$ is the Gibbs energy of mixing per lattice site, $\chi_{\text {FHS }}$ the Flory-Huggins-Staverman-interaction parameter, $\varphi_{i}$ the volume fraction of the component $i, N_{i}$ the chain length of the component $i$, and $k_{\mathrm{B}}$ is the Boltzmann constant.

In general, $\chi_{\mathrm{FHS}}$ depends on temperature $T$, pressure $p$ and volume fraction $\varphi$ of the mixture $\left(\chi_{\mathrm{FHS}}=\chi_{\mathrm{FHS}}(p, T, \varphi)\right)$. Independently of the functional form of $\chi_{\mathrm{FHS}}(p, T, \varphi)$, there are basic properties common to all binary mixtures with a miscibility gap: Firstly, the critical points are always extrema of the spinodal curve and therefore also of the coexistence curve ${ }^{4}$ and secondly, the cloud point curve where the system demixes into two phases and the coexistence curve which indicates the compositions of the two phases are identical. The phase diagram of a binary polymer mixture of mondisperse components is shown schematically in Scheme 1(a).

2. Polymer mixtures of two polydisperse components. In practice, synthesized polymers always have a molar mass distribution (polydispersity). The polydispersity can be rather
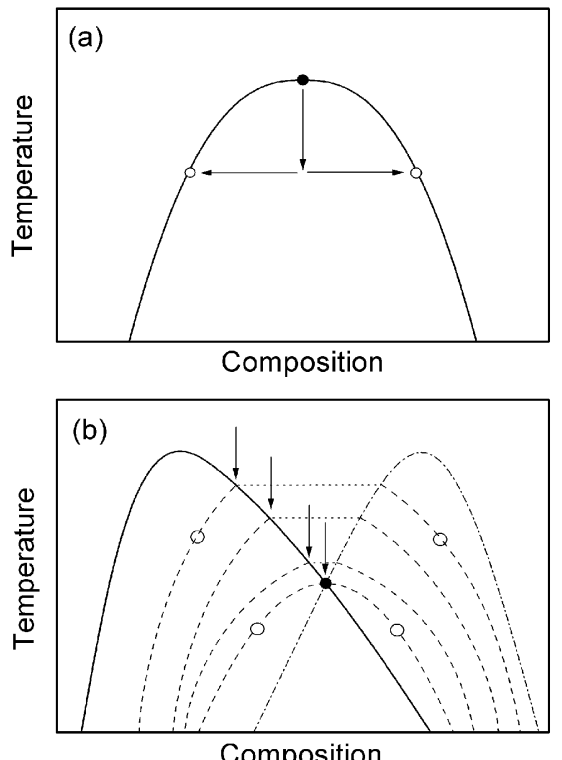

Scheme 1 (a) Schematic depiction of a phase diagram of a binary mixture. The critical point is indicated by a solid dot. The two-phase region is below the critical temperature. The curve denotes the cloud point curve which is identical to the coexistence curve of the mixture. The open dots indicate the concentrations of the two demixed phases at a certain temperature.(b) Schematic depiction of a phase diagram of a polymer mixture of two polydisperse polymers. The polymer with the higher polydispersity is on the right-hand side of the phase diagram. The solid curve depicts the cloud point curve, the dashed-dotted line the shadow curve (for an explanation of the shadow curve see text). The critical point where the cloud point curve and the shadow curve coincide is indicated by a solid dot. The dashed curves represent the coexistence curves of four mixtures of different concentrations (indicatedby the arrows). The open dots illustrate the concentrations of the demixed phases at a certain temperature for the critical mixture and one of the non-critical mixtures respectively. broad, depending on the process of polymerisation. This means that polymer mixtures are extremely high component systems with a corresponding number of degrees of freedom. Because of this, the equation for the Gibbs energy of mixing (eqn. (1)) has to be replaced by an expression that takes into account the polydispersity of the components resulting in: ${ }^{4}$

$$
\begin{aligned}
\frac{\Delta_{\mathrm{M}} G_{N}}{k_{\mathrm{B}} T} & =\sum_{j} \frac{\phi_{1 j}}{N_{1 j}} \ln \phi_{1 j}+\sum_{k} \frac{\phi_{2 k}}{N_{2 k}} \ln \phi_{2 k}+\chi_{\mathrm{FHS}} \phi_{1} \phi_{2}, \\
\phi_{1} & =\sum_{j} \phi_{1 j}, \phi_{2}=\sum_{k} \phi_{2 k}=1-\phi_{1},
\end{aligned}
$$

where $N_{1 j}\left(N_{2 k}\right)$ are the chain lengths of the chain with the molar mass $m_{j}$ in the polymer 1 (molar mass $m_{k}$ in the polymer 2) and $\varphi_{1 j}$ and $\varphi_{2 k}$ are the volume fractions of the chains of molar mass $m_{j}$ and $m_{k}$ respectively, referring to the total volume of the system.

Due to the high numbers of degrees of freedom of a system with many components, the related demixing curve is just a two-dimensional intersection through a highdimensional phase space spanned by all components. It only gives information about the total volume fractions of the two polymers. The possibility that the molar mass distributions in the two phases are different from the molar mass distributions of the pure polymers (i.e. that a fractionation occurs during demixing) is not incorporated in this information.

If the molar mass distribution of the polymers is known, the cloud point curve (demixing curve), the coexistence curve and the critical point of the mixture can be calculated numerically. ${ }^{4}$ A possibility to reduce the enormous calculation expense for a high number of components is the so-called continuous thermodynamics. ${ }^{10-13}$ This means that instead of a discrete distribution of molar masses, a continuous distribution, over which integration can be performed, is assumed. With this method, the cloud point curves, the shadow curves (for definition see below), and the critical points of different mixtures of polydimethylsiloxane and polyethylmethylsiloxane of high polydispersity could be calculated in good agreement with experimental results. ${ }^{14}$

The phase diagram of a polymer mixture of two polydisperse polymers is shown schematically in Scheme 1(b). The solid curve represents the cloud point curve of the mixture which is not identical with the coexistence curve. For each composition of the mixture in the one-phase region (shown by the location of the arrows) there exists another coexistence curve, ${ }^{15}$ represented by the dashed lines.

By cooling the mixture (the process is marked by the arrows), demixing starts at the cloud point curve where the system divides into a majority phase with approximately the composition of the mixture and an approximately infinitely small minority phase. The connection of the points of this minority phase at the temperature of demixing for different compositions of the mixture is the so-called shadow curve (dasheddotted curve in Scheme 1(b)).

The critical point, assigned by the solid dot, is not located at the maximum of the cloud point curve, but is shifted to the wing on the side of the polymer with the higher polydispersity. ${ }^{15}$ At the critical point the cloud point curve and the shadow curve intersect. The critical composition is the only composition of the mixture where no fractionation occurs by demixing, ${ }^{15,16}$ i.e. where the molar mass distributions of the polymers in the demixed phases are the same as the molar mass distributions of the pure polymers.

The phase equilibria of polydisperse systems have found recent theoretical interest, manifested through papers by Sollich et al. ${ }^{17}$ and Warren. ${ }^{18}$ For a recent review on analysing phase equilibria in polydisperse systems see Sollich. ${ }^{5}$ 


\section{B. Static light scattering}

For a critical binary polymer mixture in the vicinity of the second order phase transition, the scattered light intensity $I(\mathbf{q})$ results from the concentration fluctuations. The intensity $I(\mathbf{q})$ of the scattered light is proportional to the structure factor $S(\mathbf{q})$. For concentration fluctuations, if $q \xi$ is not too large, one gets the Ornstein-Zernike scattering law for $S(\mathbf{q})$ :

$$
S(\mathbf{q})=S(\mathbf{q}=\mathbf{0})\left(\mathbf{1}+q^{2} \xi^{2}\right)^{-1},
$$

where $\xi$ is the correlation length of the concentration fluctuations (see ref. 19).

Polymer chains have the structure of Gaussian statistical coils. The radius of gyration $R_{\mathrm{g}}$ of the Gaussian coil can be determined by light scattering with the Debye function: ${ }^{20}$

$$
S(\mathbf{q})=\frac{2}{\left(q^{2} R_{\mathrm{g}}^{2}\right)^{2}}\left(\exp \left(-q^{2} R_{\mathrm{g}}^{2}\right)-1+q^{2} R_{\mathrm{g}}^{2}\right) .
$$

If there are two contributions to light scattering in a polymer mixture, firstly the contributions of the concentration fluctuations and secondly the contributions of clusters (see Section IVB2) and assuming that the clusters have the structure of Gaussian coils, the radius of gyration of the coils $R_{\mathrm{g}}$ can be determined by a combination of eqns. (3) and (4) (see Section IVB1).

\section{Dynamic light scattering}

For polymer chains in solution it is possible to obtain a hydrodynamic radius $R_{\mathrm{H}}$ of the chains by dynamic light scattering from the Stokes-Einstein relation: ${ }^{21}$

$$
R_{\mathrm{H}}=\frac{k_{\mathrm{B}} T}{6 \pi \eta_{\mathrm{S}} D},
$$

where $\eta_{\mathrm{S}}$ is the viscosity of the solvent and $D$ the diffusion coefficient measured by dynamic light scattering. The hydrodynamic radius corresponds to the radius of a sphere which is moving through a medium of viscosity $\eta_{\mathrm{S}}$. It is proportional to the radius of gyration $R_{\mathrm{g}}$ of the chain. Experimental results for the factor of proportionality $\rho=R_{\mathrm{g}} / R_{\mathrm{H}}$ are $\rho=1.27 \pm 0.06$ for a $\theta$ solvent, ${ }^{22}$ in correspondence with results of renormalization group theory $\left(\rho=1.24,{ }^{23}\right.$ and $1.29,{ }^{24}$ respectively).

On the assumption that the clusters form Gaussian coils and that the factor of proportionality $\rho$ is the same as for a polymer in a $\theta$ solvent, it is possible to compare the results from static and dynamic light scattering.

\section{Experimental}

\section{A. Samples}

The samples were commercial products obtained from Merck (Darmstadt) and Aldrich Chemie (Stuttgart). Their molar mass distribution was measured by matrix assisted laser desorption ionisation (MALDI) mass spectroscopy. The molar mass distribution of polyethylene glycol is characterized by $M_{\mathrm{N}}=577 \mathrm{~g} \mathrm{~mol}{ }^{-1}, \quad M_{\mathrm{W}}=598 \mathrm{~g} \mathrm{~mol} \mathrm{~mol}^{-1}$, and $M_{\mathrm{W}} /$ $M_{\mathrm{N}}=1.036$. The corresponding values for polypropylene glycol are $M_{\mathrm{N}}=994 \mathrm{~g} \mathrm{~mol}^{-1}, M_{\mathrm{W}}=1019 \mathrm{~g} \mathrm{~mol}^{-1}$, and $M_{\mathrm{W}} /$ $M_{\mathrm{N}}=1.025 .{ }^{25}$ The mixtures were prepared from samples with a water content of $0.85 \%$ and $1.2 \%$ for PEG and PPG, respectively (weight fractions). These are typical values for saturated samples in ambient conditions of temperature and air humidity. The water contents were measured by Karl-Fischer titration. For the measurements, the samples were sealed.

The cloud point curve was measured with a small angle light scattering set-up described before. ${ }^{25}$ The composition of the critical mixture, $y_{\mathrm{PPG}}=0.46\left(y_{\mathrm{PPG}}\right.$ : mass fraction of polypropylene glycol), was determined by the criterion of equal volumes in the vicinity of its phase separation temperature.

\section{B. Differential refractometry}

The coexistence curves for the five investigated mixtures (mass concentrations of PPG: $y_{\mathrm{PPG}}=0.323,0.365,0.411,0.460$, 0.512 ) were determined by a differential refractometer (BricePhoenix, BP-2000-V). The samples were filtered through Millipore filters $(0.2 \mu \mathrm{m})$ into an optical cell, homogenized, and held at constant temperature in the two-phase region (below $T_{\mathrm{C}}$ ). For each differential refractometry measurement in the twophase region, the sample was left for approximately 1 day after the beginning of demixing and control measurements concerning the stability of the measurement data were made in order to exclude non-equilibrium effects.

After complete phase separation the refracted light beams for the upper and lower phase could be detected separately. The reference liquid used was a mixture of PPG and PEG with $y_{\mathrm{PPG}}=0.7$ which is homogeneous over the whole temperature range examined. In order to calculate the compositions of the demixed phases, calibration curves with a set of PEG/PPG mixtures and the pure samples were measured within a temperature range of $20^{\circ} \mathrm{C}$ to $80^{\circ} \mathrm{C}$.

\section{Light scattering experiments}

Appropriate amounts of the polymers were filtered through Millipore filters $(0.2 \mu \mathrm{m})$ into a dust-free light scattering cell. The mixtures were prepared by weight. The homogenized sample was put into the light scattering set-up and held at constant temperature until no further change in the scattered light intensity could be observed. For measurements in the twophase region below $T_{\mathrm{C}}$, macroscopic phase separation had to be reached, resulting in a typical waiting time of 1 week before each measurement. The light scattering experiments were performed with a commercial photometer (ALV, Langen). The light source was a He-Ne laser (Spectra Physics, model 127) operating at $632.8 \mathrm{~nm}$ with a power of $35 \mathrm{~mW}$. The sample was measured in an angular range $30^{\circ} \leq \theta \leq 150^{\circ}$, $150^{\circ}$, with $\theta$ being the scattering angle. This corresponds to a scattering vector range of $7.45 \times 10^{-3} \mathrm{~nm}^{-1} \leq q \leq 2.78 \times 10^{-2}$ $\mathrm{nm}^{-1}(q=(4 \pi n / \lambda) \sin (\theta / 2))$. The temperature was controlled electrically with an accuracy of $\pm 0.02 \mathrm{~K}$. The incident and the scattered laser beam were polarized perpendicular to the scattering plane (VV geometry). The scattered intensity was measured by a photomultiplier. The dynamic light scattering experiments were performed with a correlator (ALV, model 5000/E) in the same angular range as the static experiments.

\section{Results and discussion}

\section{A. Phase diagram}

In Fig. 1, the coexistence curves of five different compositions (mass fraction in the one-phase region, indicated by arrows) of the mixture PEG600/PPG1000 measured by differential refractometry are shown. They differ clearly from each other. The curves shown in Fig. 1 are fits of the equation for the order parameter $\left|y_{\mathrm{I}}-y_{\mathrm{II}}\right|=B_{0}(-\varepsilon)^{\beta}$ to the data, with the concentrations of the demixed phases $y_{\mathrm{I}}$ and $y_{\mathrm{II}}$, the reduced temperature $\varepsilon=\left(T_{\mathrm{C}}-T\right) / T_{\mathrm{C}}$ (where $T_{C}$ is also a free fit parameter), the critical exponent of the order parameter $\beta$, and the critical amplitude $B_{0}$. The results of the fitting procedures, the critical exponents and the critical amplitudes are shown as a function of the composition in the one-phase region $y_{\mathrm{PPG}}$ in Fig. 2. The lines through the data points are guides to the eyes. For the critical mixture (mass fraction of PPG: $y_{\mathrm{PPG}}=0.46$ ), the fit yielded a value of $\beta=0.357 \pm 0.008$. This 


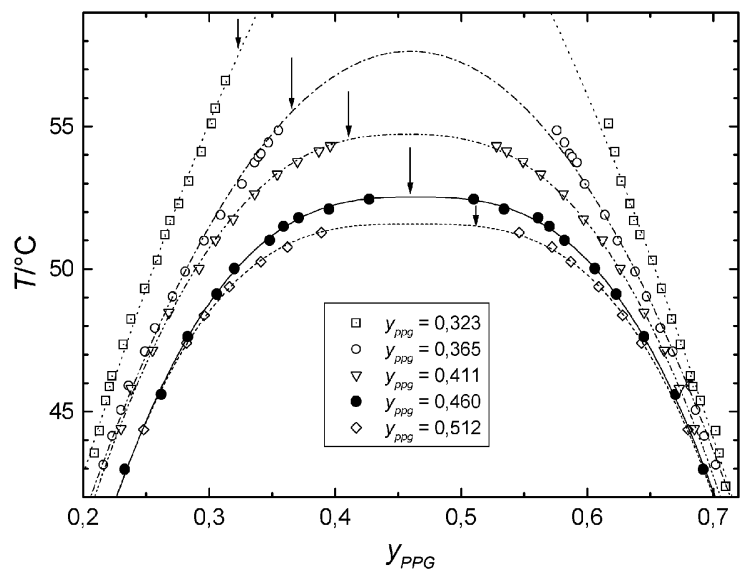

Fig. 1 Coexistence curves of five different mixtures of PEG600/ PPG1000. The compositions in the one-phase region are quoted in the box and indicated by arrows. The curves are fits of the equation for the order parameter (see text) to the data.

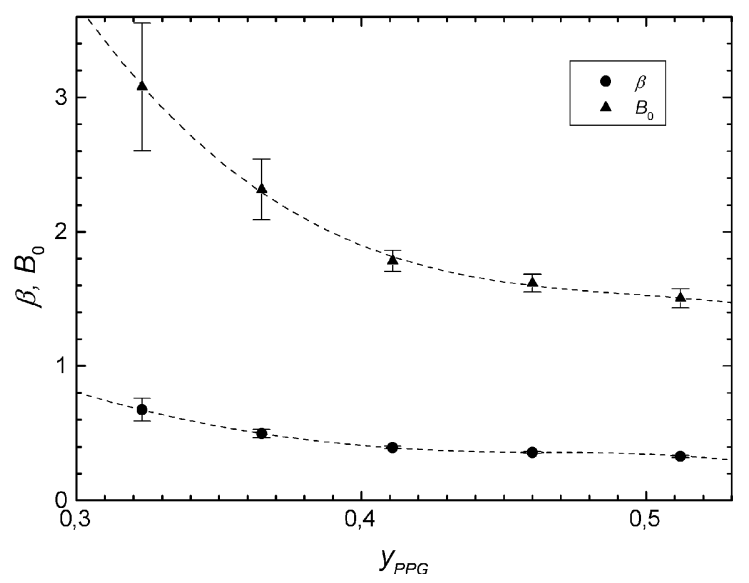

Fig. 2 Critical exponents and amplitudes of the order parameter (for the coexistence curves) from the measurements shown in Fig. 1 for all measured compositions of PEG600/PPG1000 (the mass fraction $y_{\mathrm{PPG}}$ denotes the composition in the one-phase region). The dotted lines act as guide to the eyes.

is near to the Ising value of $\beta=0.325$. The deviation for the other compositions indicate a transition to mean field behaviour $(\beta=0.5)$ for temperatures away from $T_{\mathrm{C}}$. The unphysical value of $\beta=0.67$ for $y_{\mathrm{PPG}}=0.323$ may be due to the limited number of data points for this composition. Nevertheless the set of data for the critical exponents describes a crossover from the Ising to the mean field case similar to the description of the crossover for the susceptibility as shown by Belyakov and Kiselev. ${ }^{26}$ However, there is no crossover function known for the order parameter. It would be very desirable to derive such an expression for the order parameter. According to Deutsch and Binder ${ }^{27}$ the crossover width for the order parameter should be different from that of the susceptibility. The ratio of the amplitudes $B_{0}$ for the Ising and the mean field case may contain information similar to the amplitude ratio of the susceptibility which is related to the Ginzburg number. $^{28}$

In Fig. 3, the cloud point curve of the system PEG600/ PPG1000 is shown, together with the coexistence curves. The cloud point curve was measured by small angle light scattering. The measurements are described in ref. 25. In contrast to low molecular weight binary mixtures, the cloud point curve and the coexistence curves do not coincide and the mass fraction

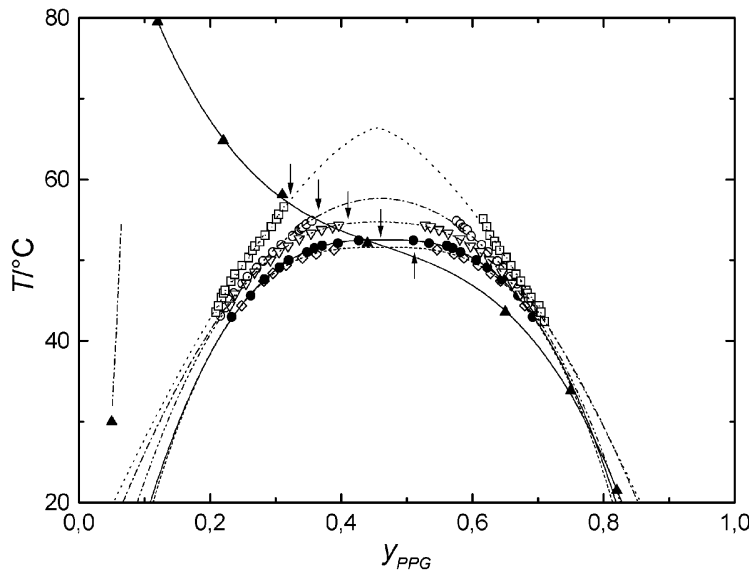

Fig. 3 Phase diagram of PEG600/PPG1000 (solid triangle: cloud point curve, solid circles and open symbols: coexistence curves, same meaning as in Fig. 1). The compositions in the one-phase region are indicated by arrows.

of the critical mixture is not equal to the composition of the mixture with the highest phase separation temperature.

We have already argued that the molar mass distribution of the samples is too narrow to explain the results (see Section IIIA). As the system has a relatively large amount of $\mathrm{OH}$ groups, the chains build clusters by intermolecular hydrogen bonding. These were measured by infrared spectroscopy in $\mathrm{PEG}^{29}$ and PPG. ${ }^{30,31}$ For PPG, ultrasound absorption ${ }^{32}$ and nuclear magnetic resonance measurements ${ }^{33}$ showed an additional low frequency relaxation process with characteristics typical for entanglement effects. The process occurred in samples with molar masses $M_{\mathrm{N}} \cong 1300 \mathrm{~g} \mathrm{~mol}^{-1}$, far below the critical molar mass of PPG for entanglements $\left(M_{\mathrm{C}} \approx 7000 \mathrm{~g}\right.$ $\mathrm{mol}^{-134}$ ). It can be explained by the reptation of hydrogen bond clusters having an effective molar mass above the critical molar mass for the onset of reptation. Further evidence for the building of clusters by hydrogen bonding comes from the fact that upon conversion of the hydroxy end groups into trimethyl silyl groups this relaxation process disappears. Thus the effect is described by a model ${ }^{35}$ which assumes the formation of "supermolecules" by intermolecular end-to-end hydrogen bonds with mean lifetimes long enough to form entanglements.

The formation of these clusters would lead to an "effective polydispersity" which can explain the difference between the cloud point curve and the coexistence curves and the difference between the critical composition and the maximum of the cloud point curve, as shown in Fig. 1 and Fig. 3. The latter result was also found for the system PEG400/PPG1000. ${ }^{36}$ As discussed above (Section IIA2), the location of the critical point on the PPG-rich side of the cloud point curve (in relation to its maximum) points to a higher polydispersity of the clusters in the PPG-rich phase.

It is interesting that the values for the concentrations of the maxima of the coexistence curves are similar for the non-critical mixtures (open symbols) and the critical mixture (solid circles), see Fig. 1 and Fig. 3. This may be taken as a hint that the additional degrees of freedom due to the fractionation of the clusters in the PEG- and PPG-rich phase allow the system to find new minima in the Gibbs energy surface.

In future investigations it would be interesting to suppress the influence of hydrogen bond clusters by using end-terminated glycols (e.g. by trimethyl silyl termination). We expect that after conversion of the hydroxy end groups the phase behaviour will be similar to that of mixtures with a small molar mass distribution and without any specific interactions. 


\section{B. Light scattering}

1. Static light scattering of the non-critical mixture in the one-phase region. In the upper part of Fig. 4, the reciprocal value of the scattering intensity of the non-critical mixture $\left(y_{\mathrm{PPG}}=0.365\right)$ is shown as a function of the square of the scattering vector $q$ (Ornstein-Zernike plot) for selected temperatures. No straight lines, as expected for Ornstein-Zernike behaviour, can be obtained in the $q$ range studied. The reason is that the scattering intensity is caused by two relevant parts (see also ref. 9 and 19): the concentration fluctuations and the clusters. For anestimation of the size of the clusters it is assumed that they have the form of Gaussian chains. Thus, a combination of the Debye function (eqn. (3)) and the Ornstein-Zernike function (eqn. (4)) was fitted to the data in order to determine the radius of gyration $R_{\mathrm{g}}$ :

$$
\begin{aligned}
I(\mathbf{q})= & K_{\text {Debye }} \frac{2}{\left(q^{2} R_{\mathrm{g}}^{2}\right)^{2}}\left(\exp \left(-q^{2} R_{\mathrm{g}}^{2}\right)-1+q^{2} R_{\mathrm{g}}^{2}\right) \\
& +K_{\mathrm{OZ}} \frac{1}{1+\xi^{2} q^{2}},
\end{aligned}
$$

where $K_{\text {Debye }}$ and $K_{\mathrm{Oz}}$ are free fit parameters which describe the contributions of the fluctuations and the clusters respectively, to the scattering intensity. In the lower part of Fig. 4, the scattering intensity is plotted as a function of the square of the scattering vector. The curves shown in the lower part of Fig. 4 are the fits of eqn. (6) to the data. Four-parameter fits were not possible, so the parameter $\xi$ was held constant. The value $\xi=10 \mathrm{~nm}$ was used which is approximately the value of the correlation length in this temperature region (see ref. 9 and 19). The mean value of the radius of gyration of the clusters obtained by this method is approximately $R_{\mathrm{g}}=(300 \pm 80) \mathrm{nm}$.

2. Dynamic light scattering. In dynamic light scattering, the autocorrelation function of the scattered intensity is measured. For the mixtures of PEG600/PPG1000, two processes could be detected, one due to the concentration fluctuations and
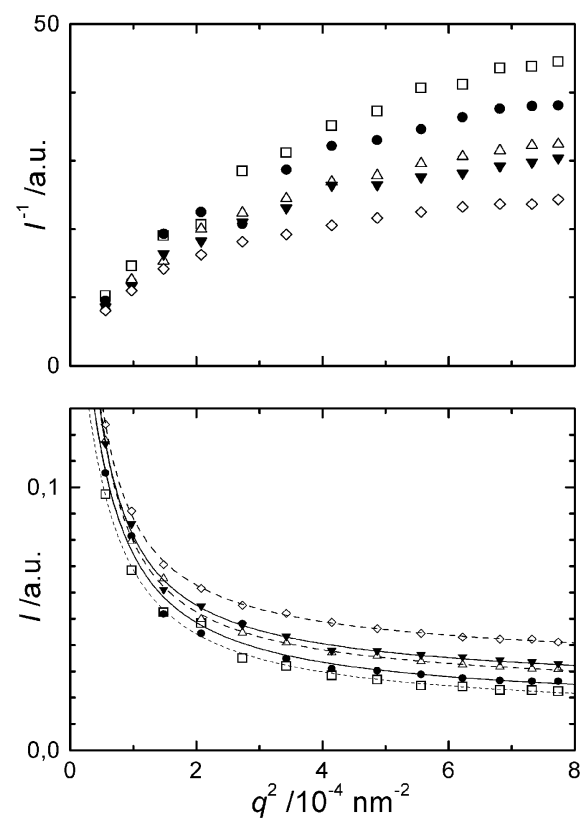

Fig. 4 Upper part: Ornstein-Zernike plot for various temperatures in the one-phase region of the non-critical mixture (open diamonds: $T=53.0^{\circ} \mathrm{C}$, solid down triangles: $T=53.4^{\circ} \mathrm{C}$, open up triangles: $T=53.6^{\circ} \mathrm{C}$, solid circles: $T=54.2^{\circ} \mathrm{C}$, open squares: $T=54.8^{\circ} \mathrm{C}$ ). Lower part: scattered light intensity as a function of $q^{2}$ for the same temperatures as in the upper part of Fig. 4.
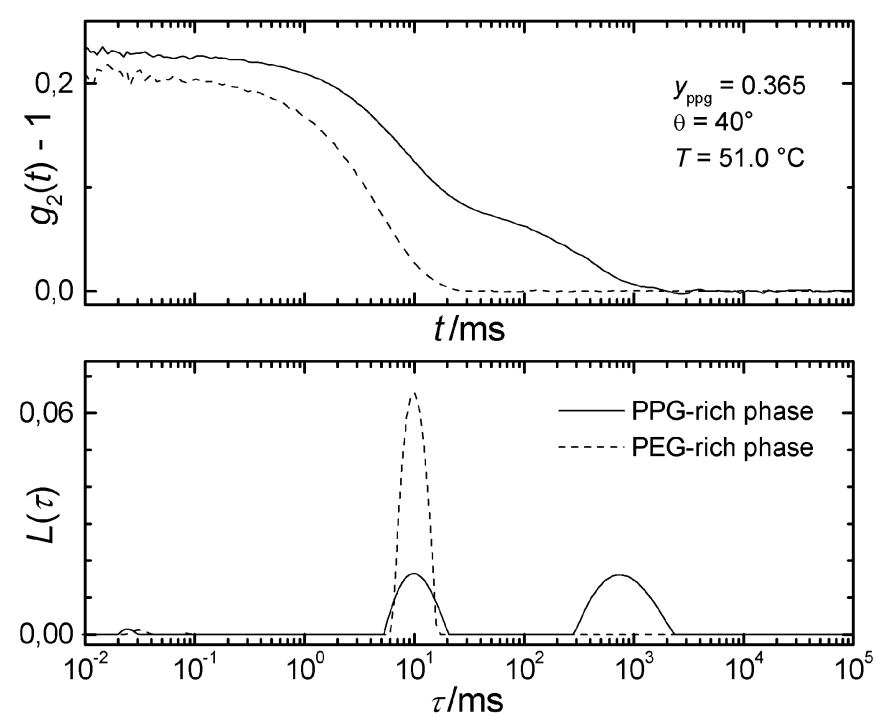

Fig. 5 Upper part: Net correlation functions of the PPG-rich and the PEG-rich phase of the non-critical mixture (solid line: PPG-rich phase, dashed line: PEG-rich phase). Lower part: corresponding distribution functions.

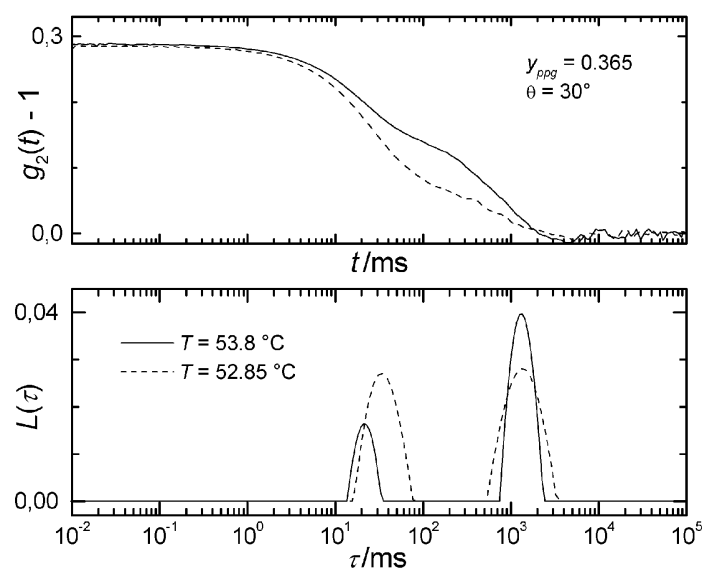

Fig. 6 Upper part: Net correlation functions of the non-critical mixture for two temperatures in the one-phase region (solid line: $T=53.8^{\circ} \mathrm{C}$, dashed line: $T=52.85^{\circ} \mathrm{C}$ ). Lower part: corresponding distribution functions.

another that could be attributed to the diffusion of hydrogen bonded clusters. The contributions of both processes depend on the composition of the mixture. Examples for a non-critical mixture ( $\left.y_{\mathrm{PPG}}=0.365\right)$ are shown in Fig. 5 and Fig. 6 . In the upper parts of the figures the net intensity correlation functions $g_{2}(t)-1$ (see ref. 19) are displayed and in the lower parts the analysis of these correlation functions with inverse Laplace transformation (using the CONTIN algorithm fitting the square of the first order correlation function) is represented.

In Fig. 5, examples for the data of the PPG-rich and the PEG-rich phase of the non-critical mixture in the two-phase region are shown. The correlation function for the PPG-rich phase (solid line) exhibits a two-step decay while in the PEG-rich phase (dotted line), only one process is seen. The obtained distribution function of relaxation times $L(\tau)$ for the PPG-rich phase clearly shows two distinct maxima where the times $\tau_{\max }$ belonging to the maxima are the inverse of the related Rayleigh linewidths $\Gamma$. The diffusion coefficient $D$ of the respective process is obtained by $D=\Gamma / q^{2}$. The faster relaxation process belongs to the concentration fluctuations of the mixture which are analysed in detail in ref. 9 and 19, 


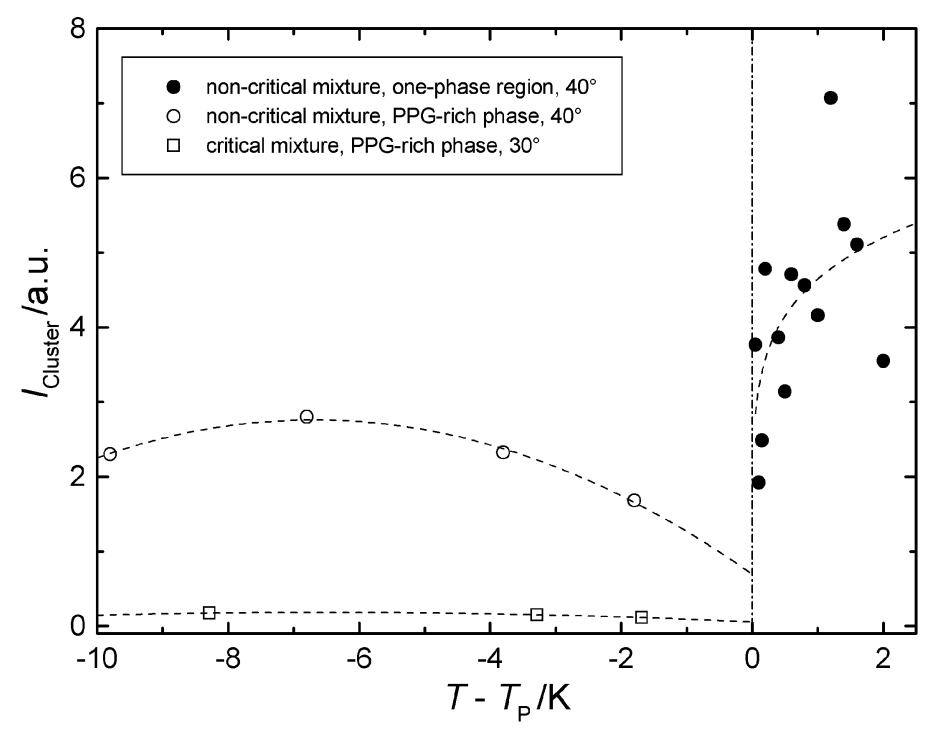

Fig. 7 Scattering intensity from the clusters as a function of the difference in the temperature of demixing. The curves through the data points act as guides to the eyes.

whereas the slower process is attributed to the diffusion of hydrogen bonded clusters. The different behaviour of the two processes can be seen in Fig. 6, where the data for two different temperatures of the non-critical mixture in the one-phase region are depicted. The times $\tau_{\max }$ of the slower process are nearly independent of temperature, while the faster process shows critical slowing down by approaching the temperature of demixing.

a Contributions of the fluctuations and the clusters to the scattering intensity. In order to get information on the amount of clusters and its dependence on the composition of the mixtures we tried to convolute the scattering intensity into the contributions from clusters and from composition fluctuations. The contributions of the clusters $I_{\text {cluster }}(\mathbf{q})$ and of the fluctuations $I_{\text {ff. }}(\mathbf{q})$ to the total scattering intensity $I(\mathbf{q})=I_{\text {fl. }}(\mathbf{q})+I_{\text {cluster }}(\mathbf{q})$ were determined by analysis of the distribution functions $L(\tau)$. The contribution of the clusters $I_{\text {cluster,rel }}$ relative to the total scattering intensity is obtained by:

$$
I_{\text {cluster }}=\frac{A_{\text {cluster }}}{A_{\text {cluster }}+A_{\mathrm{fl}}}
$$

with $A_{\text {cluster }}$ and $A_{\mathrm{fl}}$ being the areas under the peaks related to the clusters and the fluctuations respectively. Together with the total scattering intensity $I$, the absolute contribution of the clusters $I_{\text {cluster }}=I_{\text {cluster,rel }} I$ is obtained. The resulting values for $I_{\text {cluster }}$ are shown in Fig. 7 for the one-phase region of the non-critical mixture and the PPG-rich phase of the critical and non-critical mixture for scattering angles of $\theta=30^{\circ}$ and $\theta=40^{\circ}$, respectively. The curves through the data points are only guides to the eyes.

The main result of this analysis is that no scattering contributions of clusters in the PEG-rich phase could be found, neither in the non-critical mixture nor in the critical mixture. Consequently the PPG-rich phase contains considerably more hydrogen bonded clusters than the PEG-rich phase. This is in agreement with the large asymmetry in the phase diagram (see Fig. 3) which was explained by a broader distribution of the effective molar mass of the clusters in the PPG-rich phase (see Section IVA).

The values of $I_{\text {Cluster }}$ are found to be higher in the one-phase region of the non-critical mixture than in the PPG-rich phase, but no explanation can be given yet for this finding. Although the data for $I_{\text {Cluster }}$ for the non-critical mixture show a slight tendency to decrease near the temperature of demixing $T_{\mathrm{P}}$, the error limits of the $I_{\text {Cluster }}$ data do not allow us to discuss coupling of critical fluctuations to the clusters.

In the one-phase region of the critical mixture, the contribution of clusters $I_{\text {Cluster }}$ could not be detected in the scattered light intensity. This can be explained by the fact that for the critical mixture the intensity contribution due to concentration fluctuations is large and the critical slowing down of the dynamics may lead to a masking of the cluster contribution. However, the low cluster contribution in the PPG-rich phase of the critical mixture cannot be explained by this argument alone. One possible reason is a different cluster structure due to the different compositions in the two mixtures, due to different coordination numbers of the hydrogen bonds of PEG, PPG.

On the other hand, a quantitative comparison of cluster contributions to the scattered light intensity is limited since the dependence of the scattering contrast on concentration and/ or temperature is not known in detail.

$\mathrm{b}$ Size of the clusters. The hydrodynamic radius of the clusters is obtained from the diffusion coefficient by eqn. (5). It is assumed that the slow process observed by dynamic light scattering is a diffusion of clusters in an effective medium. For the viscosity of the medium in eqn. (5), the viscosity of the mixture (with its mean concentration) was used. In order to compare the results from static and dynamic light scattering, the radius of gyration of the clusters was calculated by

$$
R_{\mathrm{g}}=\rho \frac{k_{\mathrm{B}} T}{6 \pi \eta_{\mathrm{S}} D},
$$

with $\rho=1.27$ (for statistical coils in a $\theta$ solvent, see Section IIC). However, different values for $\rho$ are possible since the structure of the hydrogen bonded clusters is not known. Highly branched structures e.g. result in a different relation between hydrodynamic radius and radius of gyration.

Because of this, the absolute value of the size of the clusters obtained by eqn. (8) has to be considered with care. With $\rho=1.27$, the mean value of the radius of gyration of the clusters from dynamic light scattering is approximately $R_{\mathrm{g}}=(450 \pm 120) \mathrm{nm}$. The value from static light scattering amounts to $R_{\mathrm{g}}=(300 \pm 80) \mathrm{nm}$ and hence these values agree within experimental error. Therefore we assume that both static scattering and dynamic light scattering detect the same structures related to large clusters formed by intermolecular hydrogen bonds. 


\section{Conclusion}

Based on differential refractometry measurements we were able to determine the coexistence curves for five different compositions, including the critical one. For the latter a critical exponent of $\beta=0.357$ was found for the scaling of the order parameter, close to that for the $3 \mathrm{~d}$-Ising case $(\beta=0.325)$. Furthermore, we observed a shift of $\beta$ to the mean field value $\beta=0.5$ with increasing off-criticality.

Both the investigations on the phase behaviour and the light scattering experiments gave strong evidence for the existence of clusters built by intermolecular hydrogen bonds in the mixtures of PEG and PPG.

Although the molar mass distribution of the PEG and PPG molecules is small, the cloud point curve is asymmetric. Furthermore, the coexistence curves for different compositions in the one-phase region do not coincide and are different from the cloud point curve. These findings lead to the assumption of a broad distribution of the effective molar masses of the clusters. The assumption of hydrogen bonded clusters is in agreement with older studies on PPG. Since the maximum of the cloud point curve is shifted to small mass fractions of PPG, the "effective molar mass distribution" of the clusters in the PPG-rich phase seems to be broader than that in the PEG-rich phase. This is supported by the fact that no cluster contribution to the scattered light could be found in the PPG-rich phase, neither in the critical nor in the non-critical mixture.

Static and dynamic light scattering experiments were performed in the one- and two-phase regions of the PEG/PPG mixtures in order to get a more direct indication of the clusters. The measurements were performed separately in the PEG- and PPG-rich phase of the mixtures by changing the position of the laser beam. Both static and dynamic light scattering showed a clear indication of large structures in the range from $250 \mathrm{~nm}$ to $550 \mathrm{~nm}$. From the size and the temperature and composition dependence of the scattering contribution it is reasonable to relate these large structures to clusters built by hydrogen bonds.

The main result of this study is that oligomers with a small molar mass distribution can show a phase behaviour typical of polymers with high molar masses and broad molar mass distributions if specific interactions (here: hydrogen bonds) allow large supermolecular structures to form (at least temporarily).

The influence of intermolecular clusters on the critical composition fluctuations will be discussed in a more detailed light scattering study on this system. ${ }^{9}$ There it will be shown that the effective increase in the molar mass caused by clusters leads to a decrease in the Ginzburg number, i.e. a decrease in the temperature and composition region near the critical point where the critical fluctuations can be described in the frame of the Ising model. This again shows the similarity of the phase behaviour of large clusters formed by hydrogen bonds with the phase behaviour of polymers with a high molar mass.

\section{Acknowledgements}

The authors wish to thank Dr B. Steinhoff, L. Kühne, and Dr W. Mayer for the measurement of the cloud point curves of the mixtures. We also thank K. Rode and R. Ghahary for the characterization of the molecular weight distributions and R. Fox for the measurements of the water contents of the samples. The financial support of the Deutsche Forschungsgemeinschaft (Grant No. Al 396/1-2) is gratefully acknowledged.

\section{References}

1 (a) P. J. Flory, J. Chem. Phys., 1941, 9, 660; (b) P. J. Flory, J. Chem. Phys., 1942, 10, 51; (c) P. J. Flory, J. Chem. Phys., 1944, 12, 425.

2 (a) M. L. Huggins, J. Chem. Phys., 1941, 9, 440; (b) M. L. Huggins, J. Phys. Chem., 1942, 46, 151; (c) M. L. Huggins, J. Am. Chem. Soc., 1942, 64, 1712; (d) M. L. Huggins, Ann. N. Y. Acad. Sci., 1942, 43, 1.

3 (a) A. J. Staverman and J. H. Van Santen, Recl. Trav. Chim. PaysBas, 1941, 60, 76; (b) A. J. Staverman, Recl. Trav. Chim. PaysBas, 1941, 60, 640 .

4 R. Koningsveld, L. A. Kleintjens and H. M. Schoffeleers, Pure Appl. Chem., 1974, 39, 1.

5 P. Sollich, J. Phys.: Condens. Matter, 2002, 14, R79.

6 J. V. Sengers and J. M. H. Levelt-Sengers, in Progress in Liquid Physics, ed. C. A. Croxton, Wiley, Chichester, 1978, pp. 104-174.

7 N. Kuwahara, H. Sato and K. Kubota, Phys. Rev. Lett., 1995, 75, 1534.

8 E. C. Muniz, S. P. Nunes and B. A. Wolf, Macromol. Chem. Phys., 1994, 195, 1257.

9 S. Eckert, G. Meier and I. Alig, Phys. Chem. Chem. Phys., 2002, 4, in preparation.

10 Ch. Wolfarth and M. T. Rätsch, Kontinuierliche Thermodynamik von Copolymer-Systemen, Akademie-Verlag, Berlin, 1990.

11 J. A. Gualtieri, J. M. Kincaid and G. Morrison, J. Chem. Phys., 1982, 77, 521 .

12 J. J. Salacuse and G. Stell, J. Chem. Phys., 1982, 77, 3714.

13 J. G. Briano and E. D. Glandtl, Fluid Phase Equilib., 1983, 14, 91.

14 S. Enders, A. Stammer and B. A. Wolf, Macromol. Chem. Phys, 1996, 197, 2961.

15 M. T. Rätzsch and H. Kehlen, Prog. Polym. Sci., 1989, 14, 1.

16 G. Beaucage and R. S. Stein, Macromolecules, 1993, 26, 1603.

17 P. Sollich and M. E. Cates, Phys. Rev. Lett., 1998, 80, 1365.

18 P. B. Warren, Phys. Rev. Lett., 1998, 80, 1369.

19 S. Eckert, S. Hoffmann, G. Meier and I. Alig, Phys. Chem. Chem. Phys., 2002, 4, 2594.

20 R. Berne, R. Pecora, Dynamic Light Scattering, Wiley, Chichester, 1976.

21 W. Brown and T. Nicolai, in Dynamic Light Scattering, ed. W. Brown, Clarendon Press, Oxford, 1993, p. 277.

22 (a) W. Burchard, M. Schmidt and W. H. Stockmayer, Macromolecules, 1980, 13, 590; (b) M. Schmidt and J W. Burchard, Macromolecules, 1981, 14, 210.

23 T. Oono and M. Kohmoto, J. Chem. Phys., 1983, 78, 520.

24 J. F. Douglas and K. F. Freed, Macromolecules, 1984, 17, 2344 2354.

25 B. Steinhoff, M. Rüllmann, L. Kühne and I. Alig, J. Chem. Phys, 1997, 107, 5217.

26 M. Y. Belyakov and S. B. Kiselev, Physica A, 1992, 190, 75.

27 H. P. Deutsch and K. Binder, J. Phys. II France, 1993, 3, 1049.

28 D. Schwahn, G. Meier, K. Mortensen and S. Janßen, J. Phys. II, 1994, 4, 837 .

29 O. E. Philippova, S. I. Kuchanov, I. N. Topchieva and V. A. Kabanov, Macromolecules, 1984, 18, 1628.

30 V. N. Kuznetsov, L. V. Lesnevskaya, V. A. Petrova, V. B. Kogan and M. S. Vilesova, Polym. Sci. USSR, 1969, 11, 239.

31 B. I. Lirova, Yu. V. Latychev, N. A. Petrov, A. L. Smolyanskii and A. A. Tager, Polym. Sci. USSR, 1973, 15, 163.

32 I. Alig, S. B. Grigor'ev, Ju. S. Manučarov and S. A. Manučarova, Acta Polym., 1986, 37, 698.

33 I. Alig, E. Donth, W. Schenk, S. Höring and Ch. Wohlfarth, Polymer, 1988, 29, 2081.

34 B. A. Smith, E. T. Samulski, L- P- Yu and M. A. Winnik, Macromolecules, 1985, 18, 1901.

35 G. Heinrich, I. Alig and E. Donth, Polymer, 1988, 29, 1198.

36 W. Mayer, S. Hoffmann, G. Meier and I. Alig, Phys. Rev. E, 1997, $\mathbf{5 5}, 3102$ 